\title{
Partial recovery of disturbed V-J pairing profiles of T-cell receptor in people living with HIV receiving long-term antiretroviral therapy
}

\author{
Guoli $\mathrm{Li}^{1,2}$, Jiarui $\mathrm{Li}^{1,2}$, Henghui Zhang ${ }^{1,2}$, Yu Zhang ${ }^{3}$, Di Liu ${ }^{4,5}$, Yu Hao ${ }^{1,2}$, Junyan Han ${ }^{1,2}$, \\ Juan $\mathrm{Du}^{1,2}$, Liuluan Zhu, ${ }^{1,2}$, Yongqin Zeng ${ }^{3}$, Bei $\mathrm{Li}^{3}$, Rui $\mathrm{Li}^{1,2}$, Chuan Song ${ }^{1,2}$, Fujie Zhang ${ }^{3}$, \\ Chen Chen ${ }^{1,2^{*}}$, Hongxin Zhao $^{3^{*}}$ \& Hui Zeng ${ }^{1,2^{*}}$ \\ ${ }^{1}$ Institute of Infectious Diseases, Beijing Ditan Hospital, Capital Medical University, Beijing 100015, China; \\ ${ }^{2}$ Beijing Key Laboratory of Emerging Infectious Diseases, Beijing 100015, China; \\ ${ }^{3}$ Clinical and Research Center of Infectious Diseases, Beijing Ditan Hospital, Capital Medical University, Beijing 100015, China; \\ ${ }^{4}$ Computational Virology Group, Center for Bacteria and Virus Resources and Application, Wuhan Institute of Virology, Chinese Academy of \\ Sciences, Wuhan 430071, China; \\ ${ }^{5}$ University of Chinese Academy of Sciences, Beijing 101408, China
}

Received March 25, 2020; accepted May 16, 2020; published online June 18, 2020

\begin{abstract}
Chronic human immunodeficiency virus (HIV) infection not only causes a gradual loss of CD4 ${ }^{+} \mathrm{T}$ cells but also leads to a disturbance of the T cell receptor (TCR) repertoire. In people living with HIV (PLWH), monitoring TCR repertoire is challenged by the inconsistency of complementarity determining region 3 (CDR3) and limited cell numbers in clinical samples. Thus, a quantitative method is necessary for monitoring the TCR repertoire in PLWH.We characterized the TCR V-J pairing profile of naïve and memory $\mathrm{CD}^{+}$T cells in healthy donors, HIV-infected antiretroviral therapy (ART)-naïve patients and long-term (over 5 years) ART-experienced patients by performing TCR sequencing. We developed a V-J index with 18 parameters which were subdivided into five categories (expression coverage, cumulative percentage of the top tenth percentile, diversity, intra-individual similarity and inter-individual similarity). In ART-naïve patients, 14 of the 18 parameters were significantly altered. Long-term ART recovered ten parameters. The four unrecovered parameters were related to inter-individual similarity. Therefore, these findings indicate that long-term ART could only partially recover TCR V-J pairs and introduce newly impacted V-J pairs. Moreover, these results provide new insights into the V-J pairing of the TCR and into the disturbance of TCR repertoire in HIV infection.
\end{abstract}

acquired immune deficiency syndrome, human immunodeficiency virus, antiretroviral therapy, T cell receptor, V-J pairing

Citation: Li, G., Li, J., Zhang, H., Zhang, Y., Liu, D., Hao, Y., Han, J., Du, J., Zhu, L., Zeng, Y., et al. (2021). Partial recovery of disturbed V-J pairing profiles of T-cell receptor in people living with HIV receiving long-term antiretroviral therapy. Sci China Life Sci 64, 152-161. https://doi.org/10.1007/ s11427-020-1718-2

\section{INTRODUCTION}

$\mathrm{T}$ cells mediate immune responses to a wide variety of pa-

*Corresponding authors (Chen Chen, email: chenchen1@ccmu.edu.cn; Hongxin Zhao, email: Drzhao66@ccmu.edu.cn; Hui Zeng, email: zenghui@ccmu.edu.cn) thogenic antigens via a large repertoire of unique $\mathrm{T}$ cell receptors (TCRs) (Alcover et al., 2018; Turner et al., 2006; Turner et al., 2009). Human immunodeficiency virus (HIV) may destroy $\mathrm{CD}^{+} \mathrm{T}$ cells via virological and immunological mechanisms (Doitsh et al., 2014). Progressive loss of CD4 ${ }^{+} \mathrm{T}$ cells may further drive the TCR repertoire of $\mathrm{CD}^{+} \mathrm{T}$ cells 
away from a healthy status. The disturbed TCR repertoire of $\mathrm{CD}^{+} \mathrm{T}$ cells leads to immunosurveillance defects, resulting in opportunistic pathogen infections and tumours, namely the acquired immune deficiency syndrome (AIDS)-defining illnesses (Baum et al., 2012; Heather et al., 2015). Long-term antiretroviral therapy (ART) successfully restores $\mathrm{CD}^{+} \mathrm{T}$ cell counts in people living with HIV (PLWH). However, even after ART, the risks of certain infections and cancers, especially the non-AIDS-defining cancers, are still higher in PLWH than in the healthy population (Anglemyer et al., 2014; Deeken et al., 2012; Dominguez-Molina et al., 2016; Mocroft et al., 2010), suggesting the existence of potential defects in immunosurveillance in PLWH.

In humans, most mature $\mathrm{T}$ lymphocytes are $\mathrm{T}$ cells expressing the $\alpha \beta$ heterodimer of TCR (Alcover et al., 2018; Kreslavsky et al., 2010). The generation of TCR diversity occurs in the precursor $\mathrm{T}$ cells in the thymus by the random and imprecise somatic rearrangement of segregated germline variable $(\mathrm{V})$ and joining $(\mathrm{J})$ gene segments for the TCR $\alpha$ chain and segregated germline $\mathrm{V}$, diversity (D) and $\mathrm{J}$ gene segments for the TCR $\beta$ chain (Call et al., 2006; Mallis et al., 2015; McDonald et al., 2015). Since the hypervariable complementarity determining region 3 (CDR3) of TCRs determines the antigenic specificity of one given T cell (Call et al., 2004; Dash et al., 2017; Glanville et al., 2017), the sequences coding CDR3 have been extensively studied in the fields focusing on TCR specificity, such as anti-tumour immunity, vaccine development, and autoimmune diseases (Billam et al., 2011; Farge et al., 2017; Jiang et al., 2018; McNeel, 2016; Miyasaka et al., 2019; Ochsenreither et al., 2012; Zhao et al., 2016).

However, facing the dramatic loss of $\mathrm{CD}^{+} \mathrm{T}$ cells in PLWH, the diversity rather than the specificity is more able to indicate the severe and widespread changes in the TCR repertoire (Heather et al., 2015). The clinical usage of CDR3 has been hampered in PLWH because of the considerable genetic variation and low consistency of CDR3 among different individuals, as well as the gap between the tremendous amount of CDR3 diversity and the limited number of T cells in clinical samples (Memon et al., 2012; Wettstein et al., 2014).

Since the sequences encoding CDR3 locate at the joint of rearranged $\mathrm{V}$ and $\mathrm{J}$ segments, V-J pairing is the cornerstone of TCR diversity (Currier and Robinson, 2001; Eason et al., 2004; Venturi et al., 2008). Recently, a number of studies with immune repertoire sequencing technology revealed conservative preferential expression patterns of $\mathrm{V}$ and $\mathrm{J}$ segments in healthy individuals (Li et al., 2015; Liu et al., 2014; Migalska et al., 2018). In the present study, we aimed to quantitatively characterize the defect in TCR V-J pairing profile caused by chronic HIV infection, and the recovery of $\mathrm{T}$ cell receptors after long-term ART.

\section{RESULTS}

\section{Sequencing characteristics}

The clinical characteristics of 17 healthy donors, 17 chronically ART-naïve HIV-infected patients and 15 chronically HIV-infected ART-experienced patients are listed in Table 1. The sequenced samples of TCR $\alpha$ - and $\beta$ chains from sorted $\mathrm{T}$ cell subpopulations are summarized in Table S1 in Supporting Information. The median number of V-J paired reads was $0.95 \times 10^{5}$ per sample (range, $0.27 \times 10^{5}-$ $8.43 \times 10^{5}$ ).

\section{Healthy donors exhibited conserved preference and high similarity of V-J pairing}

The numbers of theoretical pairing types were 3,294 in the $\alpha$ chain and 1,050 in the $\beta$-chain. In healthy donors, and the V-J pairing reads covered more than $85 \%$ in the $\alpha$-chain and the $\beta$-chain of naïve and memory $\mathrm{CD}^{+} \mathrm{T}$ cells (Table 2). Heatmap analysis revealed a remarkable preference of V-J pairing in TCR $\alpha$ - and $\beta$-chains in both naïve and memory $\mathrm{CD}^{+} \mathrm{T}$ cells (Figure $1 \mathrm{~A}$ and $\mathrm{B}$ ). The cumulative percentage

Table 1 Characteristics of healthy controls, ART-naïve and ART-experienced HIV-infected patients (Median, range)

\begin{tabular}{|c|c|c|c|}
\hline & Healthy donors & ART-naïve & ART-experienced \\
\hline & $(N=17)$ & $(N=17)$ & $(N=15)$ \\
\hline Age, years & $31(23-40)$ & $29(20-43)$ & $33(25-41)$ \\
\hline Gender (Male/Female) & $17(15 / 2)$ & $17(15 / 2)$ & $15(12 / 3)$ \\
\hline Baseline $\mathrm{CD}^{+} \mathrm{T}$ cell counts (cells $\mu \mathrm{L}^{-1}$ ) & - & $318(243-443)$ & $307(212-416)$ \\
\hline $\mathrm{CD}^{+} \mathrm{T}$ cell counts (cells $\mu \mathrm{L}^{-1}$ ) & $849(556-1224)$ & $318(243-443)$ & $719(635-1112)$ \\
\hline Baseline $\mathrm{CD}^{+} \mathrm{T}$ cell counts (cells $\mu \mathrm{L}^{-1}$ ) & - & $1103(645-1963)$ & $1220(523-1618)$ \\
\hline $\mathrm{CD}^{+} \mathrm{T}$ cell counts (cells $\mu \mathrm{L}^{-1}$ ) & $772(542-1263)$ & $1103(645-1963)$ & $911(436-1950)$ \\
\hline Baseline VL $\left(\log _{10}\right.$ copies $\left.\mathrm{mL}^{-1}\right)$ & - & $4.8(4.2-5.5)$ & $4.8(4.0-5.6)$ \\
\hline pVL $\left(\log _{10}\right.$ copies $\left.\mathrm{mL}^{-1}\right)$ & - & $4.8(4.2-5.5)$ & TND \\
\hline ART duration (weeks) & - & 0 & $384(342-420)$ \\
\hline
\end{tabular}

a) pVL: plasma viral load; ART: active antiretroviral therapy; TND: the target could not be detected. 
A Naïve $\mathrm{CD}^{+} \mathrm{T}$ cells

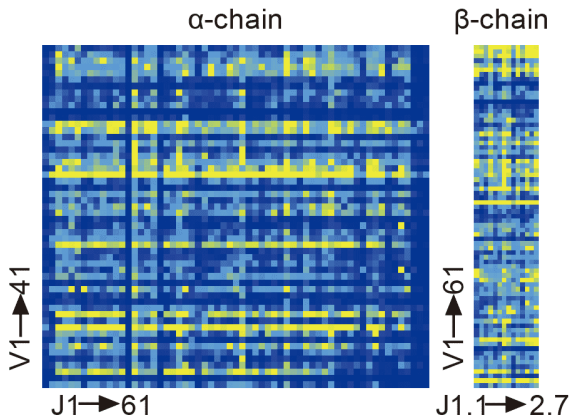

C

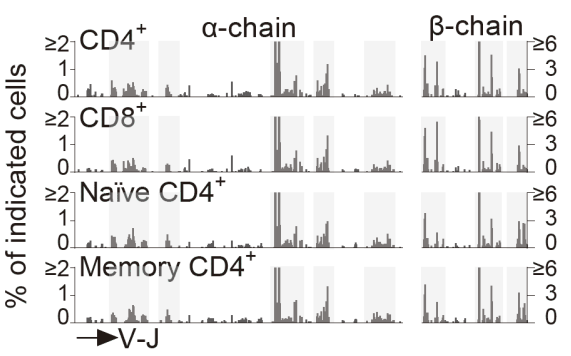

B Memory $\mathrm{CD}^{+} \mathrm{T}$ cells
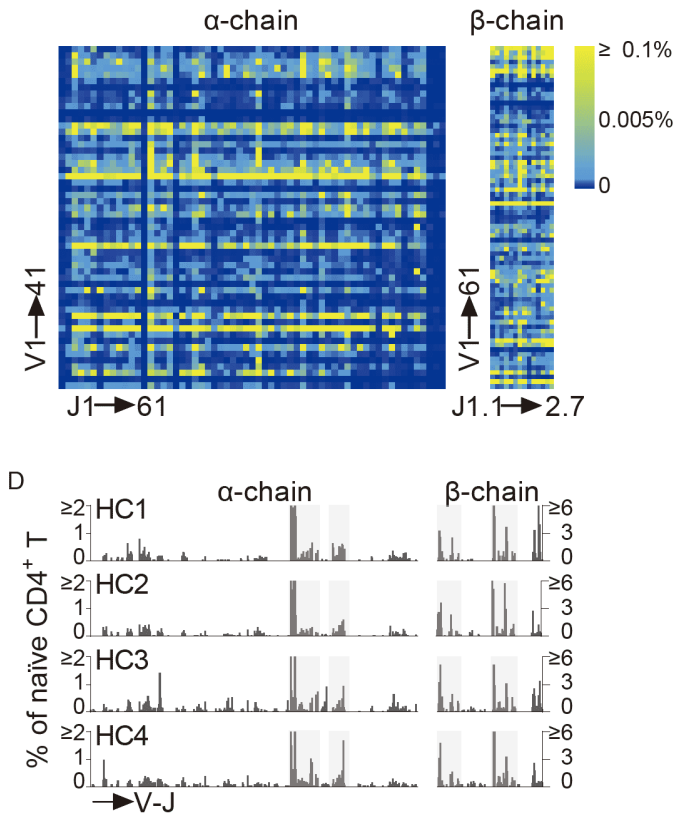

Figure 1 The preferences of TCR V-J pairs in $\mathrm{CD}^{+} \mathrm{T}$ cells of healthy donors. A and B, Heat maps of TCR $\alpha$-/ $\beta$-chain V-J pairing usages in naïve (A) and memory (B) $\mathrm{CD}^{+} \mathrm{T}$ cells. The percentages of V-J pairs are the median of healthy donors (HD). Blue and yellow represent low or high percentages of observations in the healthy donors, respectively. Colour density indicates the relative frequency level, from 0 to over $0.1 \%$. C, Similar distribution of V-J pairing profiles among different $\mathrm{CD} 4{ }^{+} \mathrm{T}$ cell subsets. The grey bar indicates the median percentages of frequency in $\alpha$ - and $\beta$-chains from different $\mathrm{T}$ cell subsets. D, Distributions of the V-J pairing frequency of naïve $\mathrm{CD}^{+} \mathrm{T}$ cell subsets in four representative individuals. The bars indicate the frequencies of presented V-J pairs in naïve CD4 ${ }^{+} \mathrm{T}$ cells. The V-J pairs with similar percentage distributions among different T cell subsets (C) or different individuals (D) are highlighted in grey.

Table 2 TCR parameters of VJ-index in Healthy donors, HIV-infected ART-naïve and experienced patients (median, range) ${ }^{\text {a) }}$

\begin{tabular}{|c|c|c|c|c|c|}
\hline VJ-index & Cell type $\& \alpha / \beta$ chain & Healthy donor & ART-naïve & ART-experienced & Recover or not \\
\hline \multirow{4}{*}{ Coverage (\%) } & Naïve $\alpha$ & $88.1(72.0-98.8)$ & $77.8(47.6-97.4)^{*}$ & $87.8(69.5-98.1)$ & $\sqrt{ }$ \\
\hline & Memory $\alpha$ & $89.9(66.5-89.9)$ & $87.8(51.7-98.0)$ & $86.7(67.8-97.0)$ & - \\
\hline & Naïve $\beta$ & $98.3(81.9-99.5)$ & $93.0(83.9-98.8)^{*}$ & $96.0(90.4-99.4)$ & $\sqrt{ }$ \\
\hline & Memory $\beta$ & $97.9(84.0-99.4)$ & $95.0(80.9-100.0)$ & $96.0(91.3-99.7)$ & - \\
\hline \multirow{4}{*}{$\begin{array}{l}\text { Cumulative frequency ( } \% \text {, } \\
\text { top } 10 \text { percentile) }\end{array}$} & Naïve $\alpha$ & $84.3(66.1-97.3)$ & $96.4(92.7-97.7)^{* * *}$ & $91.6(76.4-98.4)$ & $\sqrt{ }$ \\
\hline & Memory $\alpha$ & $85.3(64.2-98.3)$ & $96.2(86.8-97.7)^{* * *}$ & $87.0(76.4-98.6)$ & $\sqrt{ }$ \\
\hline & Naïve $\beta$ & $82.7(76.0-97.7)$ & $91.8(77.1-95.4)^{*}$ & $91.4(72.5-97.1)$ & $\sqrt{ }$ \\
\hline & Memory $\beta$ & $87.6(70.4-97.3)$ & $91.1(80.0-96.5)$ & $90.6(71.6-95.0)$ & - \\
\hline \multirow{4}{*}{ Diversity } & Naïve $\alpha$ & $5.72(3.41-6.68)$ & $4.42(3.03-5.12)^{* * *}$ & $5.47(1.78-6.21)$ & $\sqrt{ }$ \\
\hline & Memory $\alpha$ & $5.71(3.15-6.77)$ & $4.85(3.14-5.77)^{* * *}$ & $4.53(1.55-6.17)$ & $\sqrt{ }$ \\
\hline & Naïve $\beta$ & $4.81(2.64-5.25)$ & $4.17(2.93-5.10)^{*}$ & $4.54(2.90-5.84)$ & $\sqrt{ }$ \\
\hline & Memory $\beta$ & $4.64(2.76-5.43)$ & $4.09(3.54-4.98)$ & $4.19(3.15-5.35)$ & - \\
\hline \multirow{2}{*}{$\begin{array}{l}\text { Intra-individual } \\
\text { similarity }\end{array}$} & Naïve & $0.76(0.25-0.89)$ & $0.55(0.44-0.75)^{* * *}$ & $0.64(0.42-0.84)$ & $\sqrt{ }$ \\
\hline & Memory & $0.83(0.26-0.93)$ & $0.64(0.08-0.88)^{* *}$ & $0.83(0.48-0.90)$ & $\sqrt{ }$ \\
\hline \multirow{4}{*}{$\begin{array}{l}\text { Inter-individual } \\
\text { similarity }\end{array}$} & Naïve $\alpha$ & $0.47(0.09-0.82)$ & $0.33(0.13-0.66)^{* * *}$ & $0.28(0.10-0.80)^{* * *}$ & $x$ \\
\hline & Memory $\alpha$ & $0.40(0.07-0.87)$ & $0.35(0.11-0.64)^{* *}$ & $0.27(0.11-0.81)^{* *}$ & $x$ \\
\hline & Naïve $\beta$ & $0.59(0.07-0.89)$ & $0.49(0.01-0.79)^{* * *}$ & $0.30(0.07-0.85)^{* * *}$ & $x$ \\
\hline & Memory $\beta$ & $0.48(0.06-0.89)$ & $0.34(0.02-0.81)^{* * *}$ & $0.28(0.07-0.87)^{* * *}$ & $x$ \\
\hline
\end{tabular}

a) ***: $P<0.001, * *: P<0.01, *: P \leq 0.05 ; P$ values were calculated by Mann-Whitney $\mathrm{U}$ tests vs. Healthy donor. $\sqrt{ }:$ recovered in ART-experienced patients; $\times$ : unrecovered in ART-experienced patients; -: parameters were not affected by HIV infection $(P>0.05$, ART-naive vs. Healthy donor).

of the top tenth percentile accounted for approximately $85 \%$ of the total V-J pairing reads (Table 2). The top 10 most frequently used V-J pairs in the samples from healthy donors are listed in Table S2 in Supporting Information. Similar 
preferences of V-J pairing were also observed in the naïve and memory fractions of $\mathrm{CD}^{+} \mathrm{T}$ cells (Figure S1 in Supporting Information).

Of note, the V-J pairing profiles of naïve and memory fractions of $\mathrm{CD}^{+}{ }^{+} \mathrm{T}$ cells and $\mathrm{CD} 8^{+} \mathrm{T}$ cells displayed high similarity of V-J pairing profiles in each healthy donor individual (named intra-individual similarity, Figures $1 \mathrm{C}$ and S1C in Supporting Information). The similarity of V-J pairing profiles was also observed among different healthy individuals (named inter-individual similarity, Figures 1D and S1D in Supporting Information), especially in high frequency V-J pairs. Among the top 10 most frequently used V-J pairs of TCR $\alpha$ - and $\beta$-chains, the naïve and memory fractions of $\mathrm{CD}^{+} \mathrm{T}$ cells shared 8 and $9 \mathrm{~V}-\mathrm{J}$ pairs, respectively (Table S2 in Supporting Information).

Based on the conserved preferences and high similarity of the $\mathrm{V}-\mathrm{J}$ pairing profiles, we proposed 18 parameters as a $\mathrm{V}-\mathrm{J}$ pairing index to evaluate the TCR repertoire in HIV-infected patients (Table 2). These parameters cover the following five categories: (i) the coverage of V-J pairing, (ii) cumulative V$\mathrm{J}$ pairing percentage of the top tenth percentile, (iii) the diversity of V-J pairing, (iv) intra-individual similarity of V-J pairing (the similarity between naïve and memory $\mathrm{CD} 4^{+} \mathrm{T}$ cells within each individual), and (v) inter-individual similarity of V-J pairing (the similarity among individuals).

\section{Alteration of the V-J pairing index in ART-naïve PLWH}

Next, we analysed the alteration of the V-J pairing index in ART-naïve patients. The median coverage of TCR V-J pairing from naïve $\mathrm{CD}^{+} \mathrm{T}$ cells was $77.8 \%$ (range, $47.6 \%-$ $97.4 \%$ ) in the $\alpha$-chain and $93.0 \%$ (range, $83.9 \%-98.8 \%$ ) in the $\beta$-chain, which were significantly lower than those in healthy donors (median, $88.1 \%$ in the $\alpha$-chain and $98.3 \%$ in the $\beta$-chain, $P<0.05$ ) (Table 2). Meanwhile, ART-naïve patients displayed an increased preference for V-J pairing (Figure 2A) in both the $\alpha$-chain of naïve and memory $\mathrm{CD} 4^{+} \mathrm{T}$ cells and the $\beta$-chain of naïve $\mathrm{CD} 4^{+} \mathrm{T}$ cells. Consistently, the cumulative percentages of the top tenth percentile V-J pairs of ART-naïve patients were significantly higher in TCR the $\alpha$-chain $(96.4 \%)$ and $\beta$-chain $(91.6 \%)$ of naive $\mathrm{CD}^{+} \mathrm{T}$ cells and the $\alpha$-chain of memory $\mathrm{CD}^{+}$T cells $(91.8 \%)$ (Table 2$)$.

We further calculated the Shannon entropy index, which is commonly used for the measurement of diversity, and found that HIV-infected patients exhibited a significantly decreased value for V-J pairing in TCR $\alpha$ - and $\beta$-chains in naïve $\mathrm{CD} 4^{+} \mathrm{T}$ cells, as well as in the TCR $\alpha$-chain of memory $\mathrm{CD}^{+} \mathrm{T}$ cells $(P<0.05$, Figure 2B).

In agreement with the dramatic decrease in the percentage of naive $\mathrm{CD}^{+} \mathrm{T}$ cell counts (Table 1 ), we observed a decrease in intra-individual similarity in ART-naïve patients (Figure 2D). By evaluating Pearson correlation coefficients, we also found a significant reduction in inter-individual si- milarity of the TCR $\alpha$ - and $\beta$-chains in both naïve and memory $\mathrm{CD}^{+} \mathrm{T}$ cells in ART-naïve patients (Figure 2C).

As summarized in Table 2, 14 of $18 \mathrm{~V}-\mathrm{J}$ pairing parameters were significantly altered in ART-naïve HIV-infected patients.

\section{Partial recovery of the $\mathrm{V}-\mathrm{J}$ pairing index after long-term ART}

Next, we investigate whether long-term ART could successfully recover the disturbed V-J pairing index. Along with the recovery of $\mathrm{CD} 4^{+} \mathrm{T}$ cell counts, the coverage of TCR V-J pairs from naïve $\mathrm{CD}^{+} \mathrm{T}$ cells returned to $87.8 \%$ (range, $69.5 \%-98.1 \%$ ) and $96.0 \%$ (range, $90.4 \%-99.4 \%, P>0.05$, Table 2). The preferences of TCR $\alpha$ - and $\beta$-chains decreased towards healthy status (Figure $3 \mathrm{~A}$ ). In addition, the Shannon entropy diversity index of TCR $\alpha$ - and $\beta$-chain V-J pairs in $\mathrm{CD} 4^{+} \mathrm{T}$ cell subsets rose to a level comparable to healthy donors (Figure $3 \mathrm{~B}$ ). The similarity coefficients of intra-individual similarities of $\mathrm{V}-\mathrm{J}$ pairing profiles returned towards the values of healthy donors (Figure 3C). Taken together, the results of the Mann-Whitney test showed that among 14 parameters that were altered in ART-naïve patients, 10 parameters had recovered after long-term ART (Table 2). In addition, among 1,401 significantly impacted V-J pairs $(P<0.01)$ in ART-naïve patients, $1,113(79.4 \%)$ recovered after over 5 years of therapy (Figure 4$)$.

Strikingly, we observed that 4 parameters related to the inter-individual similarity coefficients of both naïve and memory $\mathrm{CD}^{+} \mathrm{T}$ cells in ART-experienced patients remained lower than those in healthy donors (Figure 3D, Table 2). All these parameters were related to the inter-individual similarity coefficients of both naïve and memory $\mathrm{CD} 4{ }^{+} \mathrm{T}$ cells, showing certain defects in the inter-individual similarity of V-J pairing profiles, in the patients, whose $\mathrm{CD} 4^{+} \mathrm{T}$ cell count recovered after long-term ART.

\section{Unrecovered and newly impacted V-J pairs after ART or HIV infection}

Next, we analysed the V-J pairs that differently expressed in ART-experienced patients and ART-naïve patients (cut-off: $P<0.01$, Figure 4). Among 705 (sum of all the V-J pairs in panel II and III of Figure 4B and C) disturbed V-J pairs in ART-experienced patients, 288 (sum of all the V-J pairs in panel II of Figure 4B and C, 40.9\%) were those unrecovered from the significantly impacted V-J pairs in ART-naïve patients. The remaining 417 (sum of all the V-J pairs in panel III of Figure 4B and C, 59.1\%) V-J pairs were comparable between healthy donors and ART-naïve patients, and became significantly different from healthy donors after ART $(P<0.01$, lower right quarter of the scatter-plots in Figure 4$)$, suggesting that the disturbance of these V-J pairs might be 

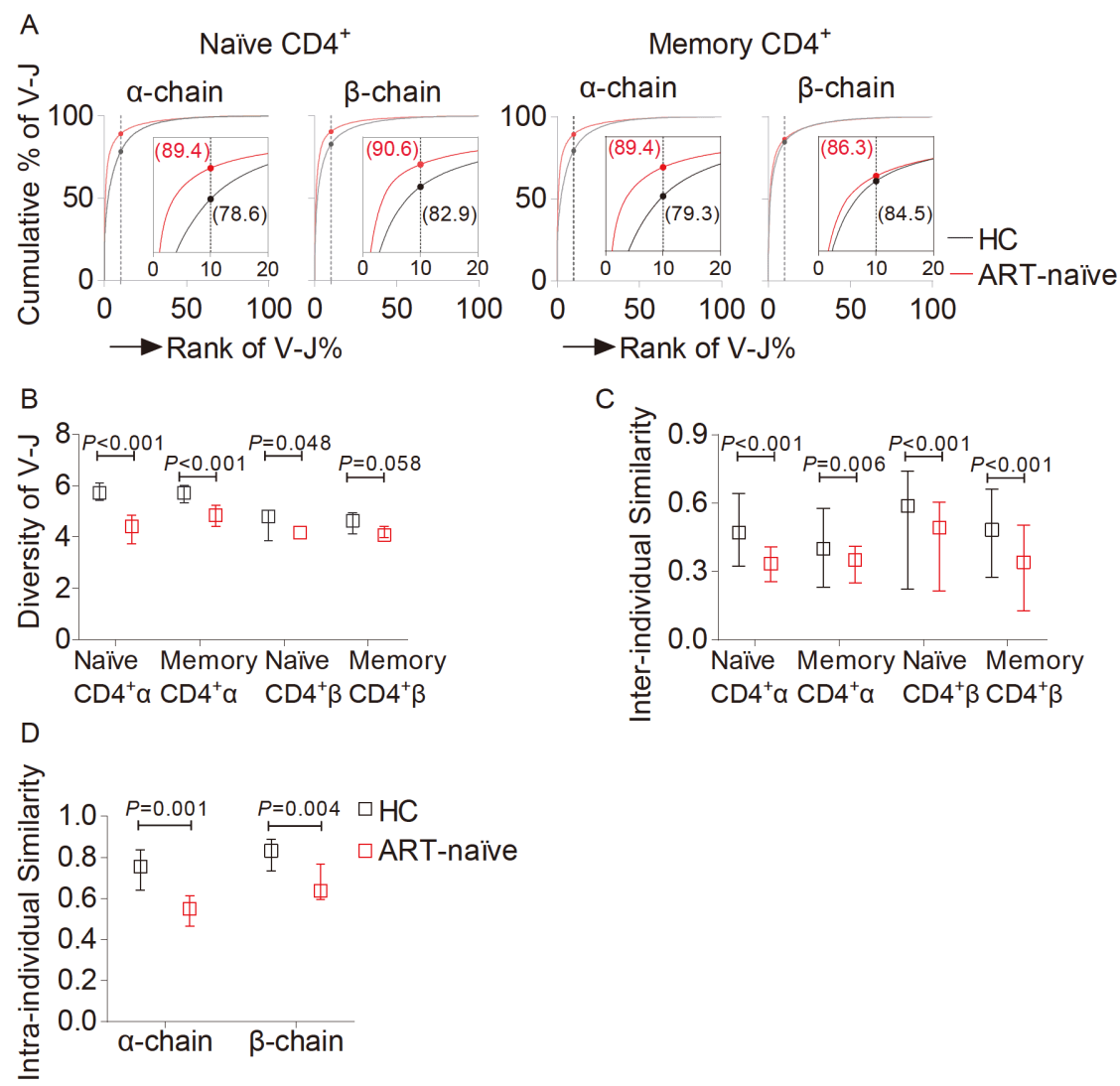

C

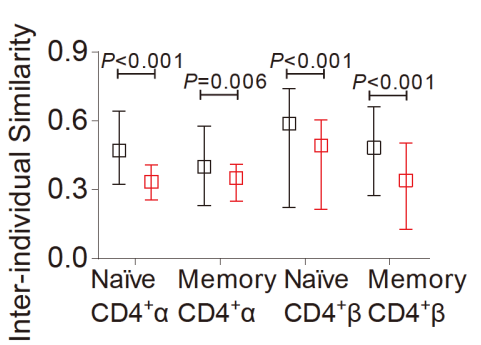

Figure 2 Preference, diversity, and intra- and inter-individual similarities of TCR V-J pairing in CD4 ${ }^{+}$T cells from HIV-infected ART-naïve patients. A, Preference of TCR V-J pairing in naïve and memory CD4 ${ }^{+} \mathrm{T}$ cells from chronic HIV infections. The curves show the accumulated frequency (median) of TCR V-J pairs presented along the rank percentage of V-J pairs in healthy donors (HD, black) and HIV-infected ART-naïve patients (red). Dashed lines and the number in brackets correspond to the cumulative percentages of the top tenth percentile of highest frequencies of V-J pairing. B, Diversities of V-J pairing in $\mathrm{CD}^{+} \mathrm{T}$ cells from patients with chronic HIV infection without ART. C, Inter-individual similarity of TCR V-J pairing in CD4 ${ }^{+} \mathrm{T}_{\text {cells }}$ from HIV-infected ART-naïve patients. D, Intra-individual similarity of TCR V-J pairing in CD4 ${ }^{+}$T cells from HIV-infected ART-naïve patients. Red boxes indicate the diversity or similarities of V-J pairs from HIV-infected ART-naïve patients, and black boxes indicate these parameters in the healthy donors. $P$ values were calculated by Mann-Whitney U tests.

introduced by ART.

Among the 288 uncovered V-J pairs, $240(83.3 \%)$ resided in the TCR $\alpha$-chain of naïve $\mathrm{CD} 4{ }^{+} \mathrm{T}$ cells, $40(13.9 \%)$ in the TCR $\alpha$-chain of memory $\mathrm{CD}^{+}$T cells, and only $5(1.74 \%)$ and $3(1.04 \%) \mathrm{V}-\mathrm{J}$ pairs were found in the TCR $\beta$-chain of naïve and memory $\mathrm{CD}^{+} \mathrm{T}$ cells, respectively (Figure $4 \mathrm{~B}$ and C). Most unrecovered V-J pairs (282 grey rings of total 288 rings in Figure 4A and B, 97.9\%) had significantly lower frequencies $(P<0.01)$ than those in healthy donors. In addition, 56.3\% (135/240) of unrecovered V-J pairs in naïve $\mathrm{CD}^{+} \mathrm{T}$ cells were concentrated into TRAV38-2/DV8 (43), TRAV8-2 (31), TRAV8-4 (26), TRAV38-1 (19) and TRAV12-1 (16), while $45.0 \%$ (18/40) of unrecovered V-J pairs in memory $\mathrm{CD} 4^{+} \mathrm{T}$ cells were concentrated into TRAV8-2 (Figures 4B and C and 5A). All these concentrated $\mathrm{V}$ segments belong to highly frequently used $\mathrm{V}$ segments in healthy donors (the rank of frequencies: TRAV8-2, 2; TRAV8-4, 4; and TRAV38-2/DV8, 11; Table S3 in Supporting Information). These $\mathrm{V}$ segments clustered in the cooccurrence networks, which suggested the relevance of their expressions (Figure 5B). By contrast, $417 \mathrm{~V}-\mathrm{J}$ pairs in the panel III of Figure 4B and C were newly impacted after ART, and they were randomly dispersed in $\mathrm{V}$ or $\mathrm{J}$ segment usage (naïve $\mathrm{CD} 4^{+} \mathrm{T}$ cell: $72 \mathrm{~V}-\mathrm{J}$ pairs increased, 48 decreased; memory $\mathrm{CD} 4^{+} \mathrm{T}$ cells: $23 \mathrm{~V}-\mathrm{J}$ pairs increased, 102 decreased; Figure $5 \mathrm{C}$ and $\mathrm{D}$ ).

\section{DISCUSSION}

In the present study, based on the conservative profile of V-J pairing of TCRs, we established a quantitative system and successfully evaluated the TCR repertoire in PLWH before and after ART. Among eighteen parameters of V-J pairing, the expression coverage, the cumulative percentage of the top tenth percentile and the diversity of V-J pairing quantitatively describe the diversity of TCR repertoire of an individual, while the intra-individual similarity and interindividual similarity of V-J pairing represent the difference in the types of TCR V-J pairing between PLWH and the 


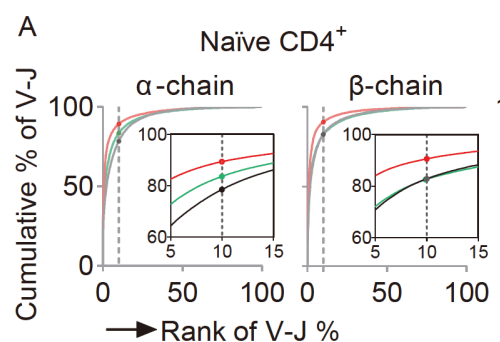

$\mathrm{B}$
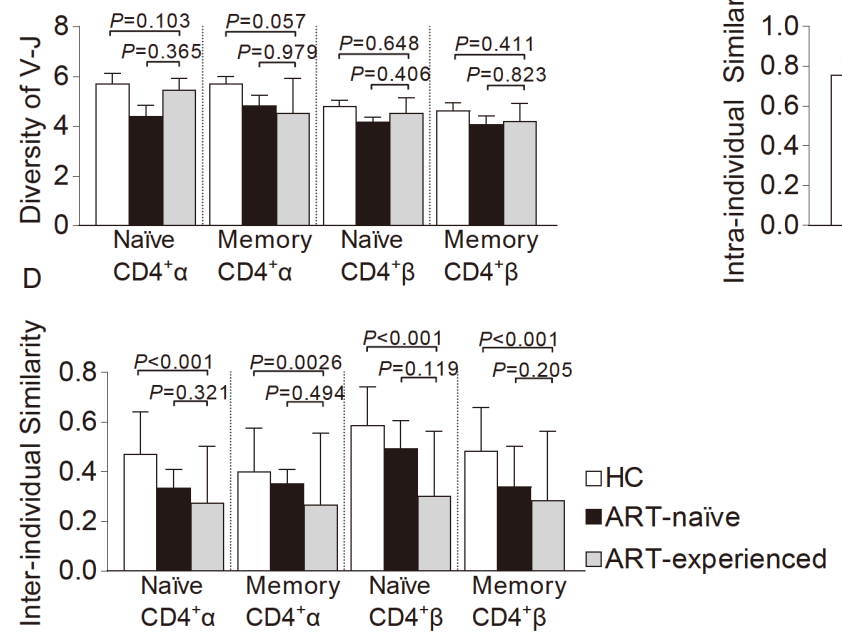

Memory CD4 ${ }^{+}$

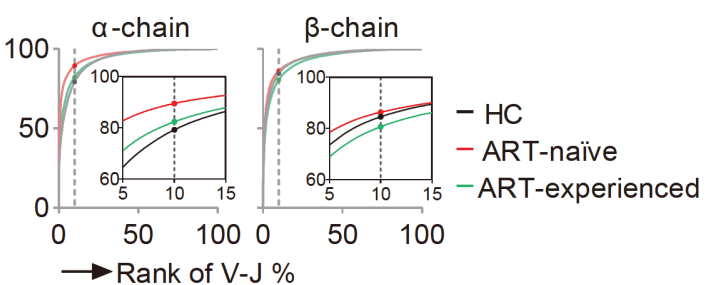

$\mathrm{C}$

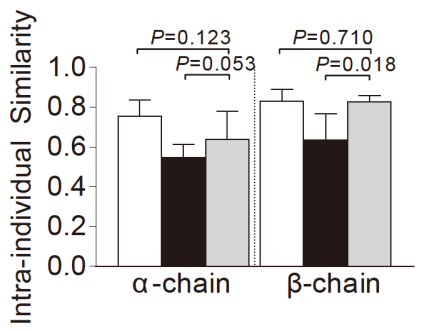

Figure 3 Changes in preference, diversity, and intra- and inter-individual similarity of $\mathrm{CD}^{+}{ }^{+} \mathrm{T}$ cells from HIV-infected ART-experienced patients. A, Preference of TCR V-J pairing in naïve and memory CD4 ${ }^{+} \mathrm{T}$ cells from HIV-infected ART-experienced patients. Black, red, and green curves show the accumulation of the percentage (median) of presented TCR V-J pairs along the rank percentage of V-J pairs in healthy donors (HD), HIV-infected ART-naïve patients and HIV-infected ART-experienced patients, respectively. B, Diversities of V-J pairing in $\mathrm{CD}^{+}{ }^{+} \mathrm{T}$ cells from HIV-infected ART-experienced patients. C, Intra-individual similarity of TCR V-J pairing in CD4 ${ }^{+}$T cells from HIV-infected ART-experienced patients. D, Inter-individual similarity of TCR V-J pairing in $\mathrm{CD}^{+} \mathrm{T}$ cells from HIV-infected ART-experienced patients. Grey bars indicate the diversity or similarities of V-J pairs from HIV-infected ARTexperienced patients. Black and white bars indicate the similarities in HIV-infected ART-experienced patients and healthy donors, respectively. $P$ values were calculated by Mann-Whitney U tests.

healthy donors. By using V-J pairing index, we explored the depressed coverage, heightened preference of total V-J pairs, increased cumulative percentages of the top tenth percentile, as well as decreased inter- and intra-individual similarities in ART-naïve patients. More importantly, although many studies have concluded that the current ART regimen may extend the life expectancy of PLWH close to that of healthy people (Wandeler et al., 2016), our data points out the limitations of the current ART regimen, i.e., partial restoration of TCR V-J pairs in $\mathrm{CD}^{+} \mathrm{T}$ cells.

To our knowledge, the only study on TCR repertoires reported by Heather and colleagues showed that short-term ART (3 months) could not restore TCR diversity and the usage patterns of V and J segments (Heather et al., 2015). However, it has been acknowledged that the increase in $\mathrm{CD}^{+} \mathrm{T}$ cell counts in blood at the early stage after ART was mainly accounted for by the emigration of $\mathrm{CD}^{+} \mathrm{T}$ cells from lymphoid organs (Bosch et al., 2006). Since it usually takes four to five years for $\mathrm{CD}^{+} \mathrm{T}$ cells to achieve a stable plateau after initiating ART (Pantazis et al., 2019), we recruited a cohort with long-term ART (more than 5 years) whose median $\mathrm{CD}^{+} \mathrm{T}$ cell counts successfully increased from a baseline of $307 \mu \mathrm{L}^{-1}$ to a plateau of $719 \mu \mathrm{L}^{-1}$. As expected, most (10/14) of the dysregulated V-J pairing indexes that were observed in ART-naïve patients shifted towards and recovered to healthy status, including the preference, coverage, diversity, and intra-individual similarity of $\mathrm{V}-\mathrm{J}$ pairs. In general, ART could partially recover preferential TCR V-J pairing in AIDS patients.

Surprisingly, long-term ART failed to recover the interindividual similarity, and the median values in ART-experienced patients even much lower than ART-naïve patients. Two factors might contribute to this phenomenon. First, approximately $20 \%$ of disturbed V-J pairs in ART-naïve patients did not reach the normal level after long-term ART. Most of these unrecovered V-J pairs resided in the naïve fraction, concentrated into several highly correlated V segments of the TCR $\alpha$-chain (TRAV38-2/DV8, TRAV8-2 and TRAV8-4) with a decreased frequency. This finding suggests that some $\mathrm{CD}^{+} \mathrm{T}$ cell subpopulations with certain $\mathrm{V}$ segments and V-J gene usage still have potential defects at the early stage of $\mathrm{T}$ cell development, which might be related 
A
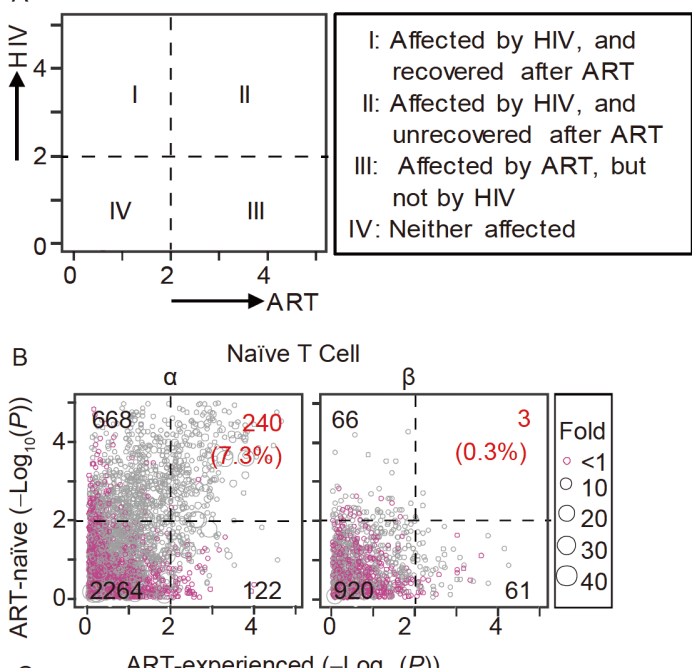

C $\quad$ ART-experienced $\left(-\log _{10}(P)\right)$

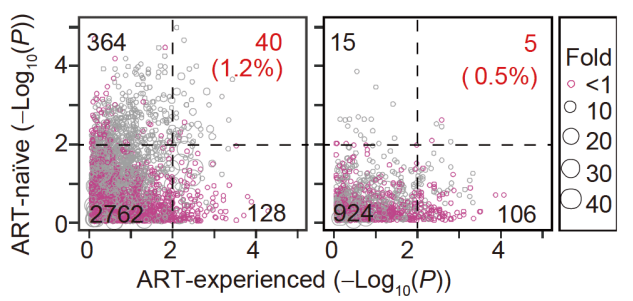

Figure 4 The V-J pairs impacted by chronic HIV infection and long-term ART. A, The schematic diagram of the V-J pairing distribution. All V-J pairs are divided into four panels with the dash representing a cut-off of $P$ value $=0.01\left(-\log _{10}(P)=2\right)$. The panel I represents the V-J pairs only impacted by HIV infections, the panel II represents the V-J pairing only impacted by ART, the panel III represents the V-J pairing impacted by both HIV infections and ART, and the left panel IV represents the V-J pairing changed neither by HIV infections nor ART. B and C, The distribution of V-J pairs of naïve (B) and memory (C) $\mathrm{CD}^{+}$T cells in patients by $P$ values during HIV infection and ART. The $x$-axis shows the $-\log _{10}(P)$ between healthy donors (HD) vs. ART-experienced patients. The $P$ value was calculated by the Mann-Whitney test. The $y$-axis shows the $-\log _{10}(P)$ between HD vs. ART-naïve patients. Red and grey rings indicate the decrease and increase in V-J frequencies in ART-experienced patients and HD, respectively. The sizes of the rings represent the fold changes in V-J pair percentage by HD/ART-experienced patients.

with some kind of pathological processes. Whether this observation is caused by residual HIV virus or local immune activation needs further investigation. Second, ART causes a more widely disturbance of V-J pairing homeostasis in both naïve and memory $\mathrm{CD}^{+}{ }^{+} \mathrm{T}$ cells, which may drives the interindividual similarity even far away from the normal status. It has been reported that zidovudine, a nucleoside analogue, can cause myelosuppression and can inhibit lymphocyte development (Rachlis and Fanning, 1993). Whether other drugs might affect TCR V-J paring requires further studies with clinical cohort or animal experiments. Minimizing these kinds of interferences to V-J pairing profiles should be taken into consideration for developing novel ART strategy.

Specificity and diversity are two important characteristics of the TCR repertoire in the adaptive immune system (Al- cover et al., 2018; Turner et al., 2006; Turner et al., 2009). Specificity describes the subtle details of $\mathrm{T}$ cell clones, whereas diversity provides a more general view of the entire $\mathrm{T}$ cell population. In most studies on the TCR repertoire, high-throughput sequencing of the CDR3 coding region has successfully described the specificity of TCR for recognizing specific peptides of pathogens, tumour cells and auto-antigens (Billam et al., 2011; Farge et al., 2017; Jiang et al., 2018; McNeel, 2016; Miyasaka et al., 2019; Ochsenreither et al., 2012; Zhao et al., 2016). Unlike the diseases related to mono- or oligo-clonal changes of $\mathrm{T}$ cells, the disturbance of $\mathrm{CD}^{+} \mathrm{T}$ cells caused by HIV infection is severe and broad (Baum et al., 2012). Thus, it is necessary to describe the changes in TCR repertoire diversity for the clinical evaluation of immune status. However, the clinical usage of the CDR3 sequence has been hindered by two factors. First, the tremendous amount of variation in the CDR3 sequences causes high inconsistency, even among healthy individuals, and leads to uncertainty in the theoretical value of CDR3 quantity (Memon et al., 2012). Second, the clinical samples represent only a very small fraction of $\mathrm{CD}^{+} \mathrm{T}$ cells in the theoretically estimated TCR repertoire based on the CDR3 sequence $\left(10^{6}\right.$ cells $\mathrm{mL}^{-1}$ vs. $10^{12}$ to $\left.10^{19}\right)$ (Alcover et al., 2018; Arstila et al., 1999; Dupic et al., 2019; Goronzy and Weyand, 2017). The V-J pairing index exhibits three advantages. First, the coverage of V-J pairing can be calculated precisely with the finite number of 3,294 V-J pairs in the $\alpha$ chain and 1,050 V-J pairs in the $\beta$-chain (Bowerman et al., 2014; Liu et al., 2014). Second, the intra-individual similarity between naïve and memory fractions may help illustrate the difference in TCR diversity of different $\mathrm{T}$ cell subsets within a single person. Third, the high inter-individual similarity allows us to successfully describe the differences in the TCR repertoire of a certain T cell subset in a given population.

Unlike previous study in which total $\mathrm{CD} 4^{+}$cells were used for TCR analysis, we investigated $\mathrm{V}-\mathrm{J}$ pairing index of naïve and memory fraction of $\mathrm{CD}^{+} \mathrm{T}$ cells. Notably, the naïve fraction of $\mathrm{CD}^{+} \mathrm{T}$ cells displayed a greater disturbance of TCR V-J pairs than the memory fraction. This finding is not surprising because HIV preferentially invades and depletes naïve $\mathrm{CD} 4{ }^{+} \mathrm{T}$ cells in blood (Rainwater-Lovett et al., 2014). The uneven distribution of naïve and memory $\mathrm{CD}^{+} \mathrm{T}$ cells also explains the decreased intra-individual similarity of V-J pairing in ART-naïve patients. Furthermore, this finding also suggested that the defects of TCR V-J pairing are not mainly mediated via HIV antigen-specific mechanisms because the naïve $\mathrm{CD} 4^{+} \mathrm{T}$ cells are not antigen-experienced.

We sequenced 314 samples of different $\mathrm{T}$ cell subsets from 49 participants, and generated about $270 \mathrm{G}$ sequencing data. The clinical sample size in this study is still limited. However, from the present exploratory research, these results may promote us to carry out a study on large clinical sample size. 

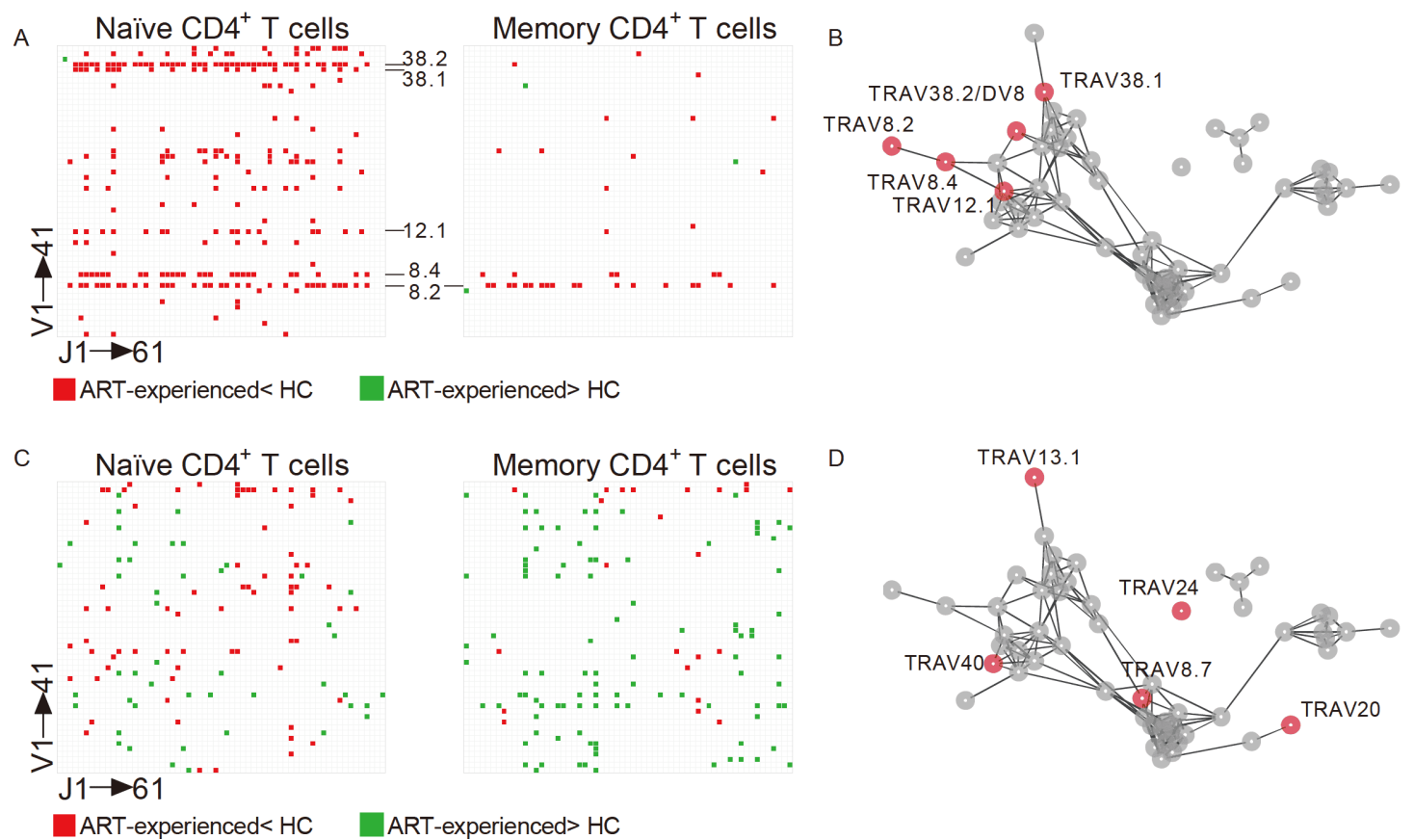

Figure 5 Characteristics of impacted TCR V-J pairs in $\mathrm{CD}^{+} \mathrm{T}$ cells after long-term ART in HIV-infected patients. A and C, Heat maps of unrecovered (A) and newly impacted (C) V-J pairs in the $\mathrm{CD}^{+} \mathrm{T}$ cell TCR $\alpha$-chain of HIV-infected ART-experienced patients. Each square in the heat maps represents a unique V-J pair. Red and green squares show healthy donors (HD) $>$ ART-experimented and HD $<$ ART-experimented percentages of V-J pairs with a cut-off $P$ value $<0.01$, respectively. The red and green squares in (A) and (C) represent the V-J pairs in the upper right panel of $\alpha$-chains from naïve and memory CD4 ${ }^{+} \mathrm{T}$ cells in Figure 4. B and D, Correlations among the highly concentrated V $\alpha$ segments of unrecovered (B) and newly impacted (D) TCR $\alpha$-chain V-J pairs in HIV-infected ART-experienced patients. Each V segment is shown as a bubble, and the line between two adjacent V segment bubbles indicates a high correlation $(>0.5)$, which was calculated by Pearson correlations. The types of unrecovered and newly impacted V-J pairs in HIV-infected ART-experienced patients were counted, and the top 5 paired $\mathrm{V} \alpha$ segments in their own section are shown in red.

It is also of interest to investigate whether the defects in TCR $\mathrm{V}-\mathrm{J}$ pairs correlate with high risks of non-AIDS-defining cancers or certain infections in PLWH after ART. We are also interested in the studies to verify the relevance between the preference of $\mathrm{V}-\mathrm{J}$ pairing and the incidence of tumors or infections. Future studies on novel long-term ART regimens are needed to improve the efficacy in recovering the TCR repertoire. In addition, we expect that the V-J pairing index may provide new quantitative parameters for clinical monitoring of the TCR repertoire in patients with other conditions causing violent disturbances of the TCR repertoire, such as sepsis, bone marrow transplantation and chemotherapy.

\section{MATERIALS AND METHODS}

\section{Study population}

The present study was approved by the Committee of Ethics at Beijing Ditan Hospital, Capital Medical University, China. All human blood samples were collected with informed consent. We recruited 17 age- and gender-paired healthy donors, 17 chronically HIV-infected ART-naïve patients, and 15 long-term (over 5 years) HIV-infected ART-experienced patients (Table 1). All HIV-infected patients (ART-naïve and -experienced patients) had baseline counts of $\mathrm{CD}^{+} \mathrm{T}$ cells between 200 and $450 \mu \mathrm{L}^{-1}$, with a median baseline plasma viral load (pVL) of $4.8 \log _{10}$ copies $\mathrm{mL}^{-1}$ (range, 4.0-5.6 $\log _{10}$ copies $\mathrm{mL}^{-1}$ ). The ART-experienced patients had a baseline of $\mathrm{CD}^{+}$T cell counts and pVL comparable to ARTnaïve patients, the level of $\mathrm{pVL}$ had been lower than 40 copies $\mathrm{mL}^{-1}$ for at least five years, and the $\mathrm{CD}^{+} \mathrm{T}$ cell counts were more than $600 \mu \mathrm{L}^{-1}$ at the time point of sample collection (Table 1).

\section{Sorting of the $\mathbf{T}$ cell subpopulation}

Fresh peripheral blood mononuclear cells (PBMCs) of all the participants were isolated with Lymphoprep ${ }^{\mathrm{TM}}$ (Stemcell, Canada). Then, the total $\mathrm{CD}^{+}$, total $\mathrm{CD} 8^{+}$, naïve and memory $\mathrm{CD}^{+} \mathrm{T}$ cells were sorted with were further sorted with magnetic beads from the MagniSort ${ }^{\mathrm{TM}}$ kits (Invitrogen, USA), including the Human CD4 naïve T Cell Enrichment Kit, the Human CD4 Memory T Cell Enrichment Kit, the Human CD4 T Cell Enrichment Kit and the Human CD8 T Cell Enrichment Kit (Ugolini et al., 2018). The purity of each sorted T cell subsets from healthy donors and ART-naïve and -experienced patients was detected by flow cytometry, and the median purities was 95.9\% (range, 92.0\% 99.6\%) (Figure S2 in Supporting Information). Approximately 
$1 \times 10^{5}$ sorted cells from each sample were used for total RNA extraction with the ReliaPrep ${ }^{\mathrm{TM}}$ miRNA Miniprep System (Promega, USA).

\section{RACE PCR, library preparation and high-throughput sequencing}

Total RNA from each sample was subjected to $5^{\prime}$ rapidamplification of cDNA ends (RACE) to obtain the unbiased amplified sequences of variable regions of both the TCR $\alpha$ and $\beta$-chains as described previously (Bowerman et al., 2014; Ozawa et al., 2008). This strategy included three continuous polymerase chain reactions (PCRs), i.e., one reverse transcription PCR (RT-PCR) and two nested PCRs. With the Superscript ${ }^{\mathrm{TM}}$ II (Invitrogen) system, the cDNA was primed by a TCR C gene segment specific primer (GSP1) and a template switching oligo-dG. The first steps of nested PCR were priming by adaptor primer 1 (AP1) and annealing of another primer (GSP2) to the $3^{\prime}$ end of GSP1, and these steps were performed with Platinum ${ }^{\mathrm{TM}} \mathrm{Pfx}$ polymerase (Invitrogen). The second nested PCR enhanced the specificity by using another adaptor primer (AP2) and annealing the other primer (GSP3) to a site located upstream of GSP2 in the $\mathrm{C}$ segment.

The PCR products were run on a $2 \%$ agarose gel, and the objective fragments in the 350-500 bp range were excised and purified using the Gel Extraction Kit (Promega). The sequencing libraries were constructed with Illumina adaptor ligation according to the manufacturer's protocol. Then, the samples were sequenced using a HiSeq 2500 platform (Illumina, USA). The sequences of each individual were generated as 250 bp paired-end reads.

\section{Statistical analysis of sequencing data}

The final TCR repertoire sequencing reads were used to identify all $\mathrm{V}$ and J gene segments of TCR $\alpha$ - and $\beta$-chains by the bioinformatics workflow of TCRklass (Li et al., 2015; Yang et al., 2015). The output data on expression percentages of each kind of V and J segment and V-J pairs, as well as their subsequent supported data, were processed by statistical analysis.

Shannon entropy indexes of chronically HIV-infected ART-experienced patients, ART-naïve patients and healthy donors were calculated to measure V-J pairing diversity. The similarity between naïve and memory $\mathrm{CD} 4^{+} \mathrm{T}$ cells within an individual was named "intra-individual similarity", and the similarity of naïve or memory $\mathrm{CD} 4^{+} \mathrm{T}$ cells among different individuals within the same population was named "interindividual similarity". These two similarities of V-J pairing preference were evaluated by Pearson correlation coefficients. The Mann-Whitney test was performed to test the differences in intra-individual similarity and inter-individual similarity among the three groups. All statistical analyses were performed using PRISM version 7 (GraphPad) and R scripts.

\section{Data availabity}

FASTQ data about the present study have been deposited in BIG (Beijing Institute of Genomics) Data Center, Chinese Academy of Sciences, accessible at https://bigd.big.ac.cn/ gsa.

Compliance and ethics The author(s) declare that they have no conflict of interest.

Acknowledgements This work was supported by the National Natural Science Foundation of China (81371804, 81772123 and 81672000), the Thirteen-fifth Key Project (2018ZX10715-005), Beijing Municipal Science and Technology Commission (Z191100006619045) and Beijing Hospital authority (DFL20191801 and DFL20191802). We thank the patients and healthy donors for their involvement in our study. We thank Xue Song at Beijing Genecast Biotechnology Co. for data analysis. We also thank Mr. Gang Wan at Beijing Ditan Hospital, Capital Medical University for valuable advice on statistics.

\section{References}

Alcover, A., Alarcón, B., and Di Bartolo, V. (2018). Cell biology of T cell receptor expression and regulation. Annu Rev Immunol 36, 103-125.

Anglemyer, A., Rutherford, G.W., Easterbrook, P.J., Horvath, T., Vitória, M., Jan, M., and Doherty, M.C. (2014). Early initiation of antiretroviral therapy in HIV-infected adults and adolescents. AIDS 28, S105-S118.

Arstila, T.P., Casrouge, A., Baron, V., Even, J., Kanellopoulos, J., and Kourilsky, P. (1999). A direct estimate of the human $\alpha \beta$ T cell receptor diversity. Science 286, 958-961.

Baum, P.D., Young, J.J., Schmidt, D., Zhang, Q., Hoh, R., Busch, M., Martin, J., Deeks, S., and McCune, J.M. (2012). Blood T-cell receptor diversity decreases during the course of HIV infection, but the potential for a diverse repertoire persists. Blood 119, 3469-3477.

Billam, P., Bonaparte, K.L., Liu, J., Ruckwardt, T.J., Chen, M., Ryder, A. B., Wang, R., Dash, P., Thomas, P.G., and Graham, B.S. (2011). T cell receptor clonotype influences epitope hierarchy in the $\mathrm{CD} 8^{+} \mathrm{T}$ cell response to respiratory syncytial virus infection. J Biol Chem 286, $4829-4841$.

Bosch, R.J., Wang, R., Vaida, F., Lederman, M.M., and Albrecht, M.A. (2006). Changes in the slope of the CD4 cell count increase after initiation of potent antiretroviral treatment. J Acquir Immune Defic Syndr 43, 433-435.

Bowerman, N.A., Falta, M.T., Mack, D.G., Wehrmann, F., Crawford, F., Mroz, M.M., Maier, L.A., Kappler, J.W., and Fontenot, A.P. (2014). Identification of multiple public TCR repertoires in chronic beryllium disease. J Immunol 192, 4571-4580.

Call, M.E., Pyrdol, J., and Wucherpfennig, K.W. (2004). Stoichiometry of the T-cell receptor - $\mathrm{CD} 3$ complex and key intermediates assembled in the endoplasmic reticulum. EMBO J 23, 2348-2357.

Call, M.E., Schnell, J.R., Xu, C., Lutz, R.A., Chou, J.J., and Wucherpfennig, K.W. (2006). The structure of the $\zeta \zeta$ transmembrane dimer reveals features essential for its assembly with the $\mathrm{T}$ cell receptor. Cell 127, 355-368.

Currier, J.R., and Robinson, M.A. (2001). Spectratype/immunoscope analysis of the expressed TCR repertoire. Curr Protoc Immunol 38.

Dash, P., Fiore-Gartland, A.J., Hertz, T., Wang, G.C., Sharma, S., Souquette, A., Crawford, J.C., Clemens, E.B., Nguyen, T.H.O., Kedzierska, K., et al. (2017). Quantifiable predictive features define 
epitope-specific T cell receptor repertoires. Nature 547, 89-93.

Deeken, J.F., Tjen-A-Looi, A., Rudek, M.A., Okuliar, C., Young, M., Little, R.F., and Dezube, B.J. (2012). The rising challenge of non-AIDSdefining cancers in HIV-infected patients. Clin Infect Dis 55, 12281235.

Doitsh, G., Galloway, N.L.K., Geng, X., Yang, Z., Monroe, K.M., Zepeda, O., Hunt, P.W., Hatano, H., Sowinski, S., Muñoz-Arias, I., et al. (2014). Cell death by pyroptosis drives CD4 T-cell depletion in HIV-1 infection. Nature 505, 509-514.

Dominguez-Molina, B., Leon, A., Rodriguez, C., Benito, J.M., LopezGalindez, C., Garcia, F., Del Romero, J., Gutierrez, F., Viciana, P., Alcami, J., et al. (2016). Analysis of non-AIDS-defining events in HIV controllers. Clin Infect Dis 62, 1304-1309.

Dupic, T., Marcou, Q., Walczak, A.M., and Mora, T. (2019). Genesis of the $\alpha \beta$ T-cell receptor. PLoS Comput Biol 15, e1006874.

Eason, D.D., Cannon, J.P., Haire, R.N., Rast, J.P., Ostrov, D.A., and Litman, G.W. (2004). Mechanisms of antigen receptor evolution. Semin Immunol 16, 215-226.

Farge, D., Arruda, L.C.M., Brigant, F., Clave, E., Douay, C., Marjanovic, Z., Deligny, C., Maki, G., Gluckman, E., Toubert, A., et al. (2017). Long-term immune reconstitution and $\mathrm{T}$ cell repertoire analysis after autologous hematopoietic stem cell transplantation in systemic sclerosis patients. J Hematol Oncol 10, 21.

Glanville, J., Huang, H., Nau, A., Hatton, O., Wagar, L.E., Rubelt, F., Ji, X., Han, A., Krams, S.M., Pettus, C., et al. (2017). Identifying specificity groups in the T cell receptor repertoire. Nature 547, 94-98.

Goronzy, J.J., and Weyand, C.M. (2017). Successful and maladaptive T cell aging. Immunity 46, 364-378.

Heather, J.M., Best, K., Oakes, T., Gray, E.R., Roe, J.K., Thomas, N., Friedman, N., Noursadeghi, M., and Chain, B. (2015). Dynamic perturbations of the T-cell receptor repertoire in chronic HIV infection and following antiretroviral therapy. Front Immunol 6, 644.

Jiang, Q., Zhao, T., Zheng, W., Zhou, J., Wang, H., Dong, H., Chen, Y., Tang, X., Liu, C., Ye, L., et al. (2018). Patient-shared TCR $\beta$-CDR3 clonotypes correlate with favorable prognosis in chronic hepatitis B. Eur J Immunol 48, 1539-1549.

Kreslavsky, T., Gleimer, M., and von Boehmer, H. (2010). $\alpha \beta$ versus $\gamma \delta$ lineage choice at the first TCR-controlled checkpoint. Curr Opin Immunol 22, 185-192.

Li, Z., Liu, G., Tong, Y., Zhang, M., Xu, Y., Qin, L., Wang, Z., Chen, X., and He, J. (2015). Comprehensive analysis of the T-cell receptor beta chain gene in rhesus monkey by high throughput sequencing. Sci Rep 5 , 10092.

Liu, P., Liu, D., Yang, X., Gao, J., Chen, Y., Xiao, X., Liu, F., Zou, J., Wu, J., Ma, J., et al. (2014). Characterization of human $\alpha \beta T C R$ repertoire and discovery of D-D fusion in TCR $\beta$ chains. Protein Cell 5, 603-615.

Mallis, R.J., Bai, K., Arthanari, H., Hussey, R.E., Handley, M., Li, Z., Chingozha, L., Duke-Cohan, J.S., Lu, H., Wang, J.H., et al. (2015). PreTCR ligand binding impacts thymocyte development before $\alpha \beta T C R$ expression. Proc Natl Acad Sci USA 112, 8373-8378.

McDonald, B.D., Bunker, J.J., Erickson, S.A., Oh-Hora, M., and Bendelac, A. (2015). Crossreactive $\alpha \beta$ T cell receptors are the predominant targets of thymocyte negative selection. Immunity 43, 859-869.

McNeel, D.G. (2016). TCR diversity-A universal cancer immunotherapy biomarker? J Immunother Cancer 4, 69.

Memon, S.A., Sportès, C., Flomerfelt, F.A., Gress, R.E., and Hakim, F.T. (2012). Quantitative analysis of $\mathrm{T}$ cell receptor diversity in clinical samples of human peripheral blood. J Immunol Methods 375, 84-92.

Migalska, M., Sebastian, A., and Radwan, J. (2018). Profiling of the TCR $\beta$ repertoire in non-model species using high-throughput sequencing. Sci Rep 8, 11613.

Miyasaka, A., Yoshida, Y., Wang, T., and Takikawa, Y. (2019). Nextgeneration sequencing analysis of the human T-cell and B-cell receptor repertoire diversity before and after hepatitis B vaccination. Hum Vaccin Immunother 15, 2738-2753.

Mocroft, A., Reiss, P., Gasiorowski, J., Ledergerber, B., Kowalska, J., Chiesi, A., Gatell, J., Rakhmanova, A., Johnson, M., Kirk, O., et al. (2010). Serious fatal and nonfatal non-AIDS-defining illnesses in Europe. J Acquir Immune Defic Syndr 55, 262-270.

Ochsenreither, S., Fusi, A., Geikowski, A., Stather, D., Busse, A., Stroux, A., Letsch, A., and Keilholz, U. (2012). Wilms' tumor protein 1 (WT1) peptide vaccination in AML patients: predominant TCR CDR3 $\beta$ sequence associated with remission in one patient is detectable in other vaccinated patients. Cancer Immunol Immunother 61, 313-322.

Ozawa, T., Tajiri, K., Kishi, H., and Muraguchi, A. (2008). Comprehensive analysis of the functional TCR repertoire at the single-cell level. Biochem Biophys Res Commun 367, 820-825.

Pantazis, N., Papastamopoulos, V., Paparizos, V., Metallidis, S., Adamis, G., Antoniadou, A., Psichogiou, M., Chini, M., Sambatakou, H., Sipsas, N.V., et al. (2019). Long-term evolution of CD4+ cell count in patients under combined antiretroviral therapy. AIDS 33, 1645-1655.

Rachlis, A., and Fanning, M.M. (1993). Zidovudine toxicity. Drug Saf 8, 312-320.

Rainwater-Lovett, K., Nkamba, H., Mubiana-Mbewe, M., Moore, C.B., Margolick, J., and Moss, W.J. (2014). Changes in cellular immune activation and memory T-cell subsets in HIV-infected Zambian children receiving HAART. J Acquir Immune Defic Syndr 67, 455-462.

Turner, S.J., Doherty, P.C., McCluskey, J., and Rossjohn, J. (2006). Structural determinants of T-cell receptor bias in immunity. Nat Rev Immunol 6, 883-894.

Turner, S.J., La Gruta, N.L., Kedzierska, K., Thomas, P.G., and Doherty, P. C. (2009). Functional implications of T cell receptor diversity. Curr Opin Immunol 21, 286-290.

Ugolini, M., Gerhard, J., Burkert, S., Jensen, K.J., Georg, P., Ebner, F., Volkers, S.M., Thada, S., Dietert, K., Bauer, L., et al. (2018). Recognition of microbial viability via TLR8 drives TFH cell differentiation and vaccine responses. Nat Immunol 19, 386-396.

Venturi, V., Price, D.A., Douek, D.C., and Davenport, M.P. (2008). The molecular basis for public T-cell responses? Nat Rev Immunol 8, 231238.

Wandeler, G., Johnson, L.F., and Egger, M. (2016). Trends in life expectancy of HIV-positive adults on antiretroviral therapy across the globe. Curr Opin HIV AIDS 11, 492-500.

Wettstein, P.J., Borson, N.D., and Kay, N.E. (2014). A novel method for analysis of human $\mathrm{T}$ cell repertoires by real-time PCR. J Immunol Methods 412, 24-34.

Yang, X., Liu, D., Lv, N., Zhao, F., Liu, F., Zou, J., Chen, Y., Xiao, X., Wu, J., Liu, P., et al. (2015). TCRklass: A new K-string-based algorithm for human and mouse TCR repertoire characterization. J Immunol 194, 446-454.

Zhao, Y., Nguyen, P., Ma, J., Wu, T., Jones, L.L., Pei, D., Cheng, C., and Geiger, T.L. (2016). Preferential use of public TCR during autoimmune encephalomyelitis. J Immunol 196, 4905-4914.

\section{SUPPORTING INFORMATION}

The supporting information is available online at https://doi.org/10.1007/s11427-020-1718-2. The supporting materials are published as submitted, without typesetting or editing. The responsibility for scientific accuracy and content remains entirely with the authors. 